\title{
DAP-MAC: A Delay-Aware Probability-based MAC Protocol for Underwater Acoustic Sensor Networks
}

\author{
Yu Han, Yunsi Fei* \\ Department of Electrical and Computer Engineering \\ Northeastern University, Boston, MA 02115
}

\begin{abstract}
The long propagation delay of underwater acoustic communications has made traditional handshaking-based medium access control (MAC) protocols inefficient for underwater sensor networks (UWSNs). We envision that this unique feature, although often taken as negative, can be leveraged to improve the parallelism between multiple senders, i.e., transmitting data simultaneously so as to achieve higher channel utilization. In this paper, we propose a novel DelayAware Probability-based MAC protocol, DAP-MAC, which eliminates the handshaking process and utilizes concurrent transmissions to significantly improve the network throughput. Compatibility relation among senders based on their distances to the common receiver is derived at run-time. A utility-optimization framework uses this compatibility relation to determine the best transmission strategies for senders. Our extensive simulations demonstrate that DAP-MAC achieves better system throughput than the existing representative underwater
\end{abstract} MAC protocols.

Keywords: Medium access control, channel access probability, compatible graph, utility optimization, underwater sensor networks.

\footnotetext{
* Corresponding author

Email addresses: yhan@ece.neu.edu (Yu Han), yfei@ece.neu.edu (Yunsi Fei)
} 


\section{Introduction}

Underwater sensor networks (UWSNs), as the enabling technology for a broad range of aquatic applications, has attracted great research interests in the past decade [1, 2]. The adverse features of underwater acoustic communication links, including long and varying propagation delay and limited and unreliable bandwidth, have rendered the design of efficient networking for UWSNs much more challenging than their terrestrial counterparts 3 .

For medium access control (MAC), coordination among sensor nodes to share the communication channel usually involves message exchange for channel reservation or consensus. Such handshaking process is widely used in terrestrial wireless networks, but is very costly in UWSNs due to the long propagation delay. For example, in conventional handshaking-based underwater MAC protocols [4, 5, 6, 7, RTS (Request To Send) and CTS (Clear to Send) control packets reserve the channel access exclusively for a pair of nodes. Their neighbor nodes hold off transmissions to avoid data collisions when they overhear the RTS or CTS packet. The exclusive access pattern of the communication channel and the large handshaking overhead make utilization of the limited bandwidth very low and also increase the packet end-to-end delay. A high-throughput MAC protocol is desirable in UWSNs.

In this work we focus on leveraging the long propagation delay of underwater acoustic communications rather than being constrained by it in networking design. The long delay actually makes certain strict requirements in RF (radio frequency)-based wireless networks unnecessary for underwater acoustic networks, such as the exclusive channel access. In RF networks without using multiplexing techniques (e.g., CDMA or FDMA), packets sent simultaneously from multiple senders to one common receiver would collide because the packet propagation time is negligible compared to the transmission time. However, in UWSNs, packets sent concurrently by different senders may arrive at one destination at different times and can be decoded separately by the receiver, considering the non-neglectable propagation time difference. 
A two-sender example is shown in Figure 1, where nodes A and B are sending packets to node $\mathrm{C}$ at the beginning of a slot in a time-slotted system. The distances between $\mathrm{A}$ and $\mathrm{C}$ and between $\mathrm{B}$ and $\mathrm{C}$ are $d_{A, C}$ and $d_{B, C}$, respectively. Assuming that packets are at length $L$ bits and the data transmission/decoding rate is $\omega$ bps, if the two distances satisfy the inequality below, the two packets sent by $\mathrm{A}$ and $\mathrm{B}$ at the same time can arrive at $\mathrm{C}$ apart without collision 1

$$
\Delta_{d}=\left|d_{A, C}-d_{B, C}\right|=\left|\left(t_{p, A}-t_{p, B}\right) \nu\right| \geq \frac{L}{\omega} \nu
$$

where $t_{p, A}$ and $t_{p, B}$ are the propagation delay of packets from node $\mathrm{A}$ to $\mathrm{C}$ and $\mathrm{B}$ to $\mathrm{C}$, respectively, and $\nu$ is the speed of sound in water (approximately 1500 $\mathrm{m} / \mathrm{s}$ ). With typical UWSN parameters $L=128$ bytes and $\omega=10 \mathrm{kbps}$, the distance difference threshold $\left(\frac{L}{\omega} \nu\right)$ for concurrent transmission is $\Delta_{\text {Thresh }}=153.6$ $\mathrm{m}$, which is attainable for distributed long-range underwater sensor networks where the transmission range reaches $2 \mathrm{~km}$. In this case, the requirement of exclusive channel occupation is too conservative for UWSNs, and the long propagation delay can allow multi-sending in a time slot to achieve higher channel utilization and system throughput. For multiple (more than two) senders, Eq.

${ }_{40}$ (1) works for each two-sender pair. If a sender's packet does not overlap with any other packets at the receiver, i.e., satisfying (1) for the sender and all other senders that transmit packets in the same time slot, the sender's packet can be successfully received.

Motivated by the inefficiency of long handshaking and the feasibility of successful concurrent transmissions in UWSNs, we design a utility-optimal delayaware probabilistic underwater random access protocol, DAP-MAC, in this paper. The proposed protocol is similar to an "Aloha" protocol with no handshaking, but each node's channel access probability is dynamically optimized at run-time in a utility-optimization framework satisfying proportional fair-

\footnotetext{
${ }^{1}$ We do not consider any capture, i.e., any overlapping packet receptions are considered as collisions and all the involved packets are discarded.
} 


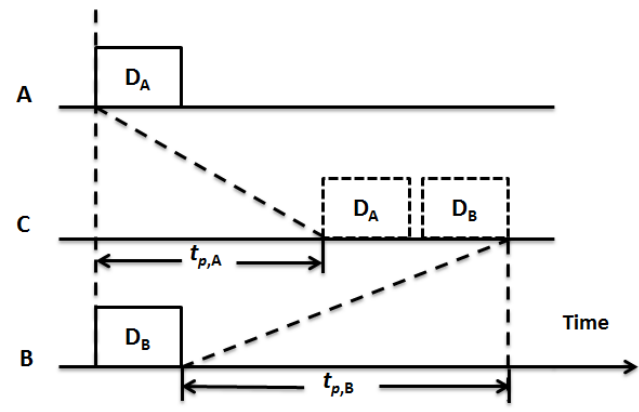

Figure 1: An example showing multi-sending, where nodes A and B send packets to node $\mathrm{C}$ at the beginning of a slot and the two packets arrive at node $\mathrm{C}$ apart without collision due to the different sender-receiver distances.

ness [8, 9, 10, 11]. Proportional fairness means allocating the channel access to senders in proportional to their packet sending success capabilities. We show theoretically that the optimal channel access probabilities are determined by the compatibility relation of senders (which will be defined later), and the optimal throughput at the receiver can be achieved by a distributed method with small control overhead. The distributive nature of DAP-MAC is suitable for long-delay and power-hungry UWSNs, which enhances the robustness further in mobile UWSNs.

The rest of the paper is organized as follows. Section 2 reviews the related work. In Section 3, we present our proposed protocol, DAP-MAC, in detail, followed by the theoretical analysis in Section 4. Performance of the protocol is evaluated in Section 5 and conclusion is given in Section 6 .

\section{Related Work}

There has emerged many medium access control protocols recently to cope with the adverse features of underwater acoustic communications. These solutions can be divided into two categories: contention-free and contention-based MAC protocols.

TDMA-based protocols, such as UW-FLASHR [12, ST-MAC [13] and STUMP [14, are typical contention-free MAC protocols for UWSNs. However, they require 
centralized scheduling, which results in either excessive control overhead or slugprotocols can be more flexible and responsive to dynamic network topologies and offered loads, and therefore are more suitable for UWSNs. This type of protocols can be further classified into handshaking-based and handshaking-free protocols.

Many handshaking-based approaches proposed for UWSNs are adapted from the traditional MACs for terrestrial networks [4, 7, 15, 6, 16, 5, 17, 18. For example, Slotted FAMA [4] and DACAP [7] both require traditional two-way handshaking prior to data transmissions. However, due to the long propagation delay in UWSNs, they achieve low channel utilization. Some approaches focus on scheduling parallel transmissions to improve channel utilization, such as APCAP [15], PDAP [6], COPE-MAC [16] and DOTS [5]. However, the large two-way handshaking overhead is a waste of channel bandwidth and therefore limits the improvement in channel utilization. Further, DOTS exhibits higher packet collisions due to missed overhearing, leading to inefficient channel usage. Other handshaking-based protocols, such as RIPT [17] and DSH-MAC [18, use the receiver-initiated approach to reduce one way of handshaking. However, the timing of initiating a packet transmission is nontrivial to determine under dynamic networks with varying traffic conditions.

Handshaking-free protocols for UWSNs, such as the simple Aloha [19, attract great attention recently for their smaller overhead and potential higher channel utilization. Several variants of Aloha-based protocols have been proposed for UWSNs 20, 21, 22, 23. Some approaches use slot guard time to reduce packet collisions [20, while some add extra control packets for collision avoidance [21]. The approaches in 22] and 23] use receiver-synchronized approach to reduce collisions in slotted Aloha. However, they all suffer from low throughput in a heavy-traffic condition. Other handshaking-free protocols, such as T-Lohi 24] and UWAN-MAC 25], have also been proposed. However, TLohi requires additional hardware to support a short tone and UWAN-MAC can only be used in delay-tolerant UWSNs. 
Our proposed protocol, DAP-MAC, is an Aloha style contention-based protocol, which eliminates the two-way handshaking for higher channel utilization. To address the possible low throughput of Aloha-type protocols due to no channel reservation [19], DAP-MAC uses a utility-optimization framework to set the optimal channel access probabilities which maximize the overall throughput. The utility-optimization framework has been widely used in networking design [9, 10, 11. We adopt the framework and formulate our MAC protocol to a utility-optimization problem considering the multi-sending capability of senders in long-delay underwater sensor networks. Such multi-sending capability utilizes the feasibility of de-synchronizing packet arrivals at the receivers under long propagation delay. De-synchronizing packet receptions has been considered in UWSNs. For example, the work in [26] proposes a geocasting solution for maximizing the coverage in the interested region, which uses different delays to prioritize packet transmissions and de-synchronize packet receptions, under an Alohatype random access setting. Our work differs from the previous work in that we leverage the long propagation delay for multi-sending, and consider a stochastic sending-probability based setting (rather than the simple Aloha), where the throughput-optimal transmission strategy (consisting of optimal channel access probabilities) is determined.

We demonstrate that the optimal proportionally fair solutions can be achieved in a distributive manner, which makes DAP-MAC a promising and robust protocol for UWSNs.

\section{DAP-MAC Protocol Design}

In this section, we first give an overview of DAP-MAC, and then provide detailed description of the protocol.

\subsection{Overview of DAP-MAC}

We focus on underwater ad hoc sensor networks where nodes can only hear their one-hop neighbors and may serve as senders or receivers for different data 
traffic.

DAP-MAC is a time-slotted contention-based protocol without handshaking. A packet can only be sent at the beginning of a slot, and a successful data transmission is followed by an ACK return. As shown in [27, 28, time synchronization among distributed nodes is achievable in underwater wireless networks, and we assume nodes follow the same slotting pattern. The slot size $T_{s}$ is chosen as the maximum propagation delay $\left(T_{\text {prop }}\right)$ plus the DATA packet transmission time $\left(T_{t x}\right)$.

A node who has a packet to send transmits the packet to the packet's destination according to its prescribed channel access probability, which is optimized at run-time by a utility-maximization framework. The channel access compatibility relation for a group, characterized by the distance difference between senders and their common receiver, is utilized to achieve parallel transmissions. We define that two nodes are channel access compatible to a common receiver if their packets can be received apart after the nodes transmit packets concurrently. Intuitively, senders with more compatible nodes should be given higher probabilities, and vice versa. By explicitly exploring concurrent transmissions and assigning optimal probabilities for channel access, DAP-MAC is able to efficiently utilize the channel in long-delay UWSNs. Implemented in a distributive manner, DAP-MAC also handles network mobility very well.

\subsection{The Utility-Optimization Framework}

DAP-MAC uniquely takes into account the group compatibility relation and adopts a utility-optimization framework to determine the optimal channel access probabilities. We start from utility optimization for a simple network, which consists of a common receiver and a set of senders. We will show that this approach requires only a little additional information exchange which is feasible for UWSNs, and it improves the system performance significantly.

For a set of senders $\mathcal{N}^{c}=(1, \ldots, N)$ of a receiver $c$, each sender $i, i \in \mathcal{N}^{c}$, is associated with a channel access probability $p_{i}^{c} \in \mathcal{P}_{i}^{c}=[0,1]$, and utility function $u_{i}^{c}: \mathcal{P}^{c}=\times_{i \in \mathcal{N}^{c}} \mathcal{P}_{i}^{c} \rightarrow \mathbb{R}$. The utility function $u_{i}^{c}$ can be represented 
using the throughput (i.e., packet success rate) achieved by sender $i$, which is characterized by the compatible graph in DAP-MAC, as defined below.

Definition 1. (Compatible Graph) In an undirected compatible graph $G^{c}=$ $(V, E)$ with respect to node $c, V$ contains all c's neighbor nodes, and for each edge $(u, v) \in E$ nodes $u$ and $v$ are compatible, for $\left(u^{\prime}, v^{\prime}\right) \notin E$ nodes $u^{\prime}$ and $v^{\prime}$ are not compatible.

We define the compatible set of node $i, \mathcal{C}_{i}^{c}$, consisting of all its compatible nodes to receiver $c$ and itself, and the interference set $\mathcal{I}_{i}^{c}$ as the complementary set of $\mathcal{C}_{i}^{c}$, i.e., $\mathcal{I}_{i}^{c}=\mathcal{N}^{c} \backslash \mathcal{C}_{i}^{c}$. Further, we define the Compatibility Factor (CF) of node $i$ as the number of elements in set $\mathcal{C}_{i}^{c},\left|\mathcal{C}_{i}^{c}\right|$, and the Interference Factor $(I F)$ as $\left|\mathcal{I}_{i}^{c}\right|$.

As seen from Figure 1, two senders are compatible if the difference of their distances to the receiver is larger than a threshold. The threshold is defined as $\Delta_{\text {Thresh }}=\left(\frac{L}{\omega}+\delta\right) \nu=\left(T_{t x}+\delta\right) \nu$, where $\delta$ is a small amount of time added to account for node mobility or fluctuation in propagation delay [29, 30]. As an example, Figure 2b depicts a compatible graph for a star network (shown in Figure 2a) with the receiver $c$ and 5 senders. With a distance threshold of $160 \mathrm{~m}$, nodes 1,3 and nodes 1,4 are two compatible pairs. The compatible set of sender 1 with respect to receiver $c$ contains nodes 1,3 and 4 with $\left|\mathcal{C}_{1}^{c}\right|=3$, and its interference factor $\left|\mathcal{I}_{1}^{c}\right|=2$. For an arbitrary compatible graph, we will show in Section 4 that the mean of compatibility factor for a receiver-centered subnetwork varies with the number of senders, maximum transmission range and distance threshold, and maintains a high value for a typical set of underwater network parameters. A high compatibility factor indicates a high chance of successful concurrent transmissions, which is the design basis for DAP-MAC.

Based on the compatibility relation, the packet success rate of sender $i$, as the throughput indicator, is given by:

$$
u_{i}^{c}=p_{i}^{c} \prod_{j \in \mathcal{I}_{i}^{c}}\left(1-p_{j}^{c}\right)
$$




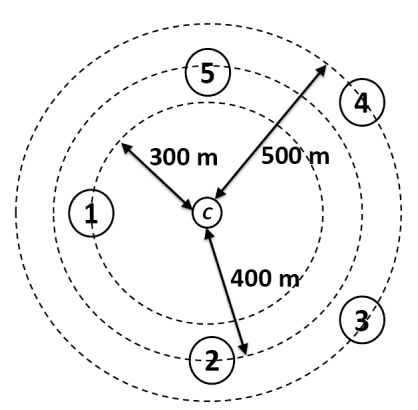

(a)

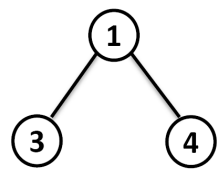

(2)

(b)

Figure 2: An example star network with the receiver's compatible graph. (a) The 5-sender 1-receiver star network topology. (b) The compatible graph of receiver $c$.

which is the probability that when node $i$ transmits a packet with probability $p_{i}^{c}$, all nodes in node $i$ 's interference set do not send packets in the same slot. Under the proportional fairness constraint [8], the optimal channel access probability vector $\mathrm{p}^{c *}=\left\{p_{1}^{c *}, p_{2}^{c *}, \ldots, p_{N}^{c *}\right\}$ can be obtained by maximizing the objective function $U^{c}(\mathrm{p})=\sum_{i \in \mathcal{N}^{c}} \log \left(u_{i}^{c}\right)$, with $p_{i}^{c} \in[0,1], \forall i \in \mathcal{N}^{c}$. We have the following theorem.

Theorem 1. For the utility function given by (2), the optimal proportionally fair channel access probability for node $i$ with respect to the receiver $c$ is given by

$$
p_{i}^{c *}=\frac{1}{1+\left|\mathcal{I}_{i}^{c}\right|}
$$

Proof 1. We omit the receiver index c for conciseness. According to (2), the objective function $U(p)=\sum_{i \in \mathcal{N}} \log \left(p_{i}\right)+\sum_{i \in \mathcal{N}} \sum_{j \in \mathcal{I}_{i}} \log \left(1-p_{j}\right)$. Since $\log (x)$ and $\log (1-x)$ are both strictly concave functions of $x, U(p)$ is strictly concave of $p$ and therefore has a unique global maximum. By taking the derivative of $U(p)$ with respect to $p_{i}$, we have $\frac{\partial U(p)}{\partial p_{i}}=\frac{1}{p_{i}}-\frac{\left|\mathcal{I}_{i}\right|}{1-p_{i}}$, where $\left|\mathcal{I}_{i}\right|$ is node $i$ 's interference factor, also the number of terms $\log \left(1-p_{i}\right)$ appearing in $U(p)$. With $\left.\frac{\partial U(p)}{\partial p_{i}}\right|_{p^{*}}=0$, we have $p_{i}^{*}=\frac{1}{1+\left|\mathcal{I}_{i}\right|}$.

Theorem 1 establishes the optimal channel access probabilities for senders that achieve the maximum overall packet success rate (i.e., the throughput) 
at the receiver. The values of those optimal probabilities only depend on the interference factors of the senders. The higher the value of IF, the lower the access probability should be, due to a higher chance of packet collisions. In the case that no node is compatible with any other node, i.e., $\left|\mathcal{I}_{i}\right|=N-1$, the optimal access probability $p_{i}^{*}=1 / N, \forall i \in \mathcal{N}$, which is the established result for RF-based networks where the propagation delay is negligible. The result in Theorem 1 also implies that DAP-MAC can run in a distributive manner as long as the senders know the values of their IFs. We will show in Section 3.3 that in DAP-MAC, the values of IFs are broadcast by the receiver upon any change of its compatible graph, with very small communication cost.

\subsection{Neighbor Discovery and Compatibility Relation Update}

Nodes discover neighbors via periodically broadcasting HELLO messages, which are widely used in ad hoc networks for building local connectivity [31, in addition to received or overheard packets. When a packet is sent, the time stamp is also included in the header. When a node receives or overhears a packet, it can calculate the propagation delay (and also the distance) between itself and the packets sender. Each node maintains an active neighbor table which includes neighbor IDs and their distances to the node. Based on the neighbor table, each node, as a potential receiver, is able to set up the channel access compatible graph of its group, as described in Section 3.2 .

A node updates its compatible graph whenever there is any change on the topology of the group, for example, a neighbor leaving, a new neighbor joining, or distance changes because of node movements. Upon detecting the compatibility relation change, the node has to notify its neighbors (potential senders). We design a small control packet, UPDATE, for this usage. It contains the ID of the node (receiver) and the interference factor for each neighbor in the receiver's group. The receiver transmits an UPDATE packet either by piggybacking it to ACK packets when an ACK is scheduled, or when the UPDATE packet is about to expire (the elapsed time since UPDATE is generated reaches an upper limit Tupdate). 


\subsection{Data Transmission Strategy}

Figure 3 depicts the transmission timeline in DAP-MAC with two senders (A and $\mathrm{B}$ ) and one receiver $(\mathrm{C})$.

As a sender, a node keeps a list of optimal channel access probabilities to all its neighbors (receivers). Whenever the node realizes a change on its interference factor to a neighbor via the neighbor's UPDATE packet, it updates the new optimal probability to the neighbor according to Theorem 1. When a sender has a DATA packet to send (the head-of-line (HOL) packet in the data queue), it chooses the action of transmit or wait at the beginning of a slot according to the channel access probability to the packet's receiver. After sending a DATA packet, the node keeps listening to the channel for an ACK packet from the receiver. A packet collision is detected when the node does not receive an ACK from the receiver within two time slots since it sends the packet, or the overheard ACK does not contain the sender's ID. Then the sender initiates a retransmission according to the prescribed access probability in the following slots. The number of retransmissions is bounded by a maximum retransmission number $\left(L_{\max }\right)$ to avoid standstill of the data queue due to adverse communication links.

As a receiver, if a node successfully receives DATA packets (may be from multiple senders) in the current slot, it will send an ACK packet in the next slot immediately acknowledging reception of those packets. Other pending DATA packets are transmitted later after the ACK.

The pseudo codes for implementing the transmission strategy of a node (e.g., node $i$ ) in DAP-MAC are shown in Algorithm 1, where we use curState and preState to indicate the current state and the previous state of a node, respectively. Since a node can be both a sender and a receiver, it has three possible states: IDLE, WAITACK and ACK. A node is initialized with an IDLE state and is allowed to send a DATA packet only when it is in IDLE state. Whenever it sends a DATA packet, it switches to the WAITACK state to wait for an ACK packet arriving in the next slot. If it receives a DATA packet destined for it in either IDLE or WAITACK state, it changes to the ACK state and prepares 


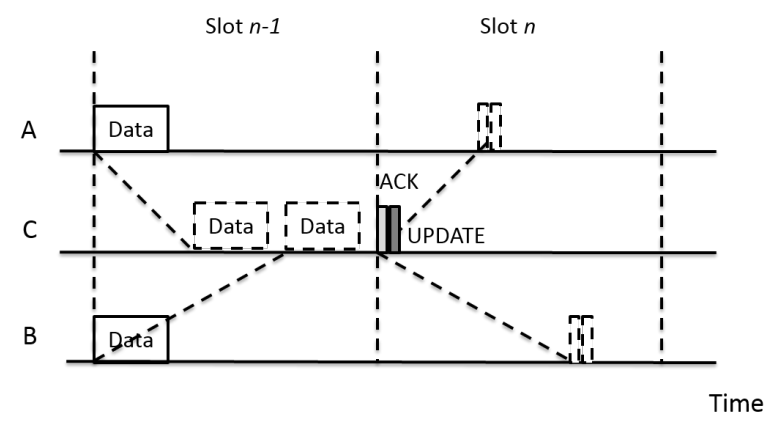

Figure 3: An example transmission timeline of DAP-MAC, where two senders $\mathrm{A}$ and $\mathrm{B}$ send packets to receiver $\mathrm{C}$ in slot $n-1$ and the receiver $\mathrm{C}$ replies an ACK packet with a piggybacked UPDATE packet in slot $n$.

to send an ACK packet with a piggybacked UPDATE packet (if any) at the beginning of the next slot. After sending the ACK packet, the node will switch back to its previous state (IDLE or WAITACK). It is worth noting that we give higher sending priority to an ACK packet over a new DATA packet, to favor the links associated with the already transmitted but unacknowledged packets.

\subsection{Mobility Support}

Our DAP-MAC protocol is adaptive to network mobility and can provide robust services in dynamic underwater environment. As shown in previous sections, nodes are capable of capturing the changes of distances between themselves and their neighbors, updating the compatibility relations of their groups, and notifying their neighbors of such changes by sending UPDATE packets.

In addition, when nodes move, the sender-receiver distance difference of two compatible senders may reduce, which possibly makes them incompatible after the change. Since the group compatibility relation is detected at one time and the receiver generates an UPDATE packet, it may take at most $T_{\text {update }}+$ $T_{\text {prop }}$ time for the neighbor nodes to recognize this relation change and send packets with their new prescribed channel access probabilities. During this time, the two senders may still send packets using unnecessarily higher access probabilities, leading to more packet collisions. Therefore, in order to guarantee 


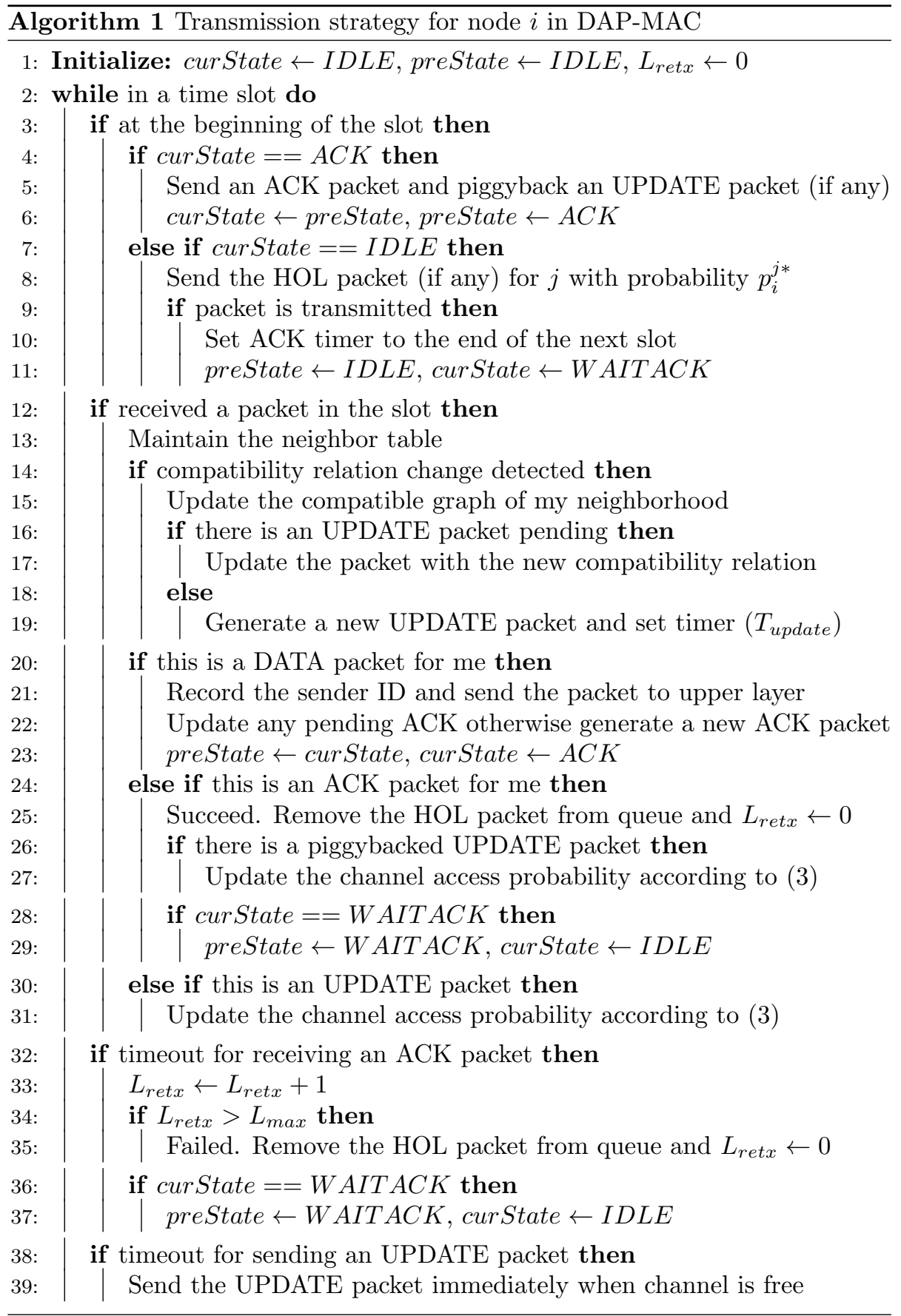


that senders use the correct channel access probabilities, we add a guard time

$\delta$ to the distance difference threshold, $\Delta_{\text {Thresh }}$, to further improve robustness. Assume the average node moving speed is $\zeta$. The guard time can be set to $\delta=\frac{2 \zeta\left(T_{\text {update }}+T_{\text {prop }}\right)}{\nu}$, where $\nu$ is the sound speed in water.

\section{Analysis}

In this section, we first study the statistical mean of the compatibility factor, a measure of the potential for concurrent transmission in DAP-MAC. Then we give the optimal network throughput of DAP-MAC to show how the optimal transmission strategy achieves higher throughput with the compatibility relation considered.

\subsection{Statistics on Compatibility Factor}

We consider the scenario that $N$ senders are independently and identically distributed (i.i.d.) in $\left(0, d_{\max }\right)$ with respect to a common receiver, where $d_{\max }$ is the characterized maximum transmission range of the acoustic modem. Let $f(x)$ be the probability distribution function (PDF) of the sender-receiver distances. We first focus on two senders and calculate the probability that they are compatible. Then we extend our analysis to multiple senders to obtain the mean of compatibility factor.

Let $X_{1}$ and $X_{2}$ be two random variables representing the individual distances between the two senders and their receiver, with $f_{X_{1} X_{2}}\left(x_{1}, x_{2}\right)$ as their joint $\mathrm{PDF}$. Then the cumulative distribution function ( $\mathrm{CDF}$ ) of their sender-receiver distance difference, i.e., $Z=\left|X_{1}-X_{2}\right|$, can be represented by $F_{Z}(z)=P\left(\mid X_{1}-\right.$ $\left.X_{2} \mid \leq z\right)=\int_{0}^{d_{\max }} d X_{2} \int_{x_{2}-z}^{x_{2}+z} f_{X_{1} X_{2}}\left(x_{1}, x_{2}\right) d X_{1}$. As two senders are defined to be compatible with each other if and only if their distance difference to the receiver is larger than a distance threshold $\Delta$, we can obtain the probability that the two senders are compatible by 32 ]

$$
P_{c}=1-F_{Z}(\Delta)=1-\int_{0}^{d_{\max }} d X_{2} \int_{x_{2}-\Delta}^{x_{2}+\Delta} f_{X_{1} X_{2}}\left(x_{1}, x_{2}\right) d X_{1} .
$$


This two-sender compatibility probability is also the probability that an observed sender has one compatible colleague from the rest of $(N-1)$ senders. With the i.i.d. assumption of the sender-receiver distance distribution, the event that any sender is compatible with the observed sender is an independent event, which occurs with the probability $P_{c}$. According to the definition of binomial distribution [32], the probability that $m$ senders from the $(N-1)$ senders are compatible with the observed sender follows the binomial distribution, $B\left(N-1, P_{c}\right)$. Such $m$-sender compatibility probability can be expressed by 32

$$
P_{c}^{m}=\left(\begin{array}{c}
N-1 \\
m
\end{array}\right)\left(P_{c}\right)^{m}\left(1-P_{c}\right)^{N-1-m} .
$$

Note that the term $N-1$ counts the number of senders (potential competitors) excluding the observed sender.

Recall that the compatible set for a sender includes the sender itself, we finally obtain the mean of compatibility factor for a set of $N$ senders as

$$
E[\mathrm{CF}]=1+\sum_{m=0}^{N-1} m \cdot P_{c}^{m}=1+(N-1) P_{c},
$$

where $P_{c}$ is given in (4). The variance of the compatibility factor can be given by $\sigma^{2}[\mathrm{CF}]=(N-1) P_{c}\left(1-P_{c}\right)$.

We next take an example to evaluate the mean of CF numerically. Assume that the sender-receiver distance follows a uniform distribution, i.e., $f(x)=$ $1 / d_{\max }, x \in\left(0, d_{\max }\right)$. According to (4), we can calculate $P_{c}$ as $P_{c}=\left(d_{\max }-\right.$ $\Delta)^{2} / d_{\text {max }}^{2}$. Substituting $P_{c}$ in (6), we obtain the mean of compatibility factor as

$$
E[\mathrm{CF}]=1+\frac{(N-1)\left(d_{\max }-\Delta\right)^{2}}{d_{\max }^{2}} .
$$

We see that the mean of $\mathrm{CF}$ varies with the number of senders $(N)$, the maximum transmission range $\left(d_{\max }\right)$ and the distance threshold $(\Delta)$. Figure 4 depicts how the mean of $\mathrm{CF}$ changes with each factor while the other two keep fixed. It can be seen that under some cases a sender can be compatible with many 


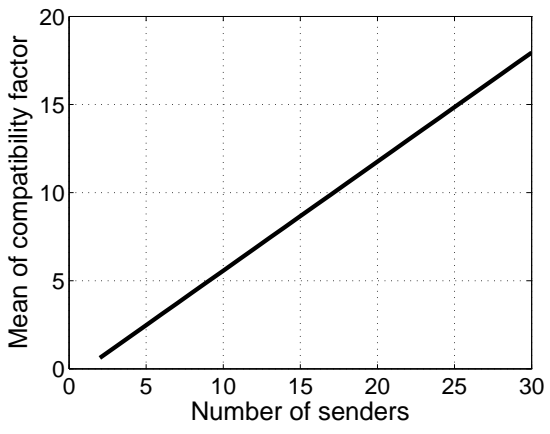

(a) $E[\mathrm{CF}]$ with $N$.

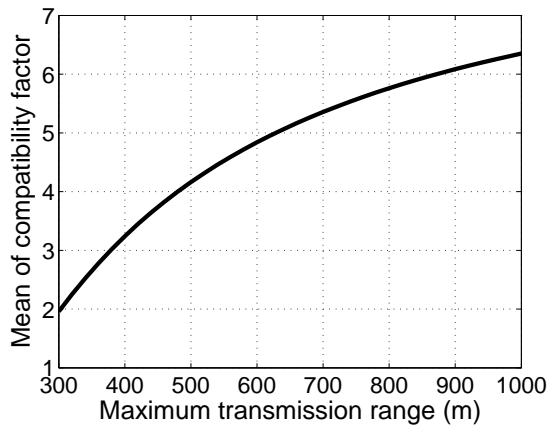

(b) $E[\mathrm{CF}]$ with $d_{\max }$.

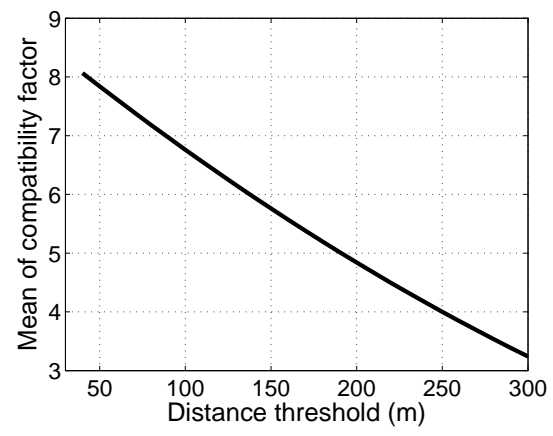

(c) $E[\mathrm{CF}]$ with $\Delta$.

Figure 4: $E[F]$ changes with the number of senders $(N)$, the maximum transmission range $\left(d_{\max }\right)$ and the distance threshold $(\Delta)$, under a typical set of underwater parameters $\left(N=10, d_{\max }=750 \mathrm{~m}\right.$ and $\left.\Delta=160 \mathrm{~m}\right)$.

other senders. For example, given a typical set of underwater parameters with $N=10, d_{\max }=750 \mathrm{~m}$ and $\Delta=160 \mathrm{~m}, E[\mathrm{CF}]$ equals approximately 5.5 , indicating that a sender can successfully transmit its packets when transmitting simultaneously with another five more senders. The higher compatibility factor, the higher probability that DAP-MAC is able to schedule successful concurrent transmissions. Our proposed protocol fully takes advantage of such parallel sending opportunities to efficiently utilize the channel bandwidth, and therefore is capable of increasing the network throughput significantly. 


\subsection{The Optimal Network Throughput}

In this section, we first analyze the optimal network throughput of DAPMAC. Then, we compare DAP-MAC to $p$-persistent slotted Aloha (S-ALOHA) [19] to show how the optimal channel access probabilities in DAP-MAC improve the throughput.

We consider a network composed by a couple of senders and a common receiver and assume a saturated condition where senders always have packets to send. With the optimal access probabilities obtained according to Theorem 1 and the definition of the utility in (2), the optimal network throughput in packets per second can be represented by

$$
S^{*}=\frac{1}{T_{s}} \sum_{i} u_{i}^{*}=\frac{1}{T_{s}} \sum_{i} p_{i}^{*} \prod_{j \in \mathcal{I}_{i}}\left(1-p_{j}^{*}\right)
$$

which is the sum of each individual throughput achieved by the senders normalized by the slot duration. The total packet success rate $\left(\sum_{i} u_{i}^{*}\right)$, as part of the throughput, is contributed by two components: the success rate of single transmissions $\left(P_{\text {single }}\right)$ and the success rate of concurrent transmissions $\left(P_{\text {concu }}\right)$, which are expressed as

$$
\begin{aligned}
P_{\text {single }} & =\sum_{i} p_{i}^{*} \prod_{j \neq i}\left(1-p_{j}^{*}\right), \\
P_{\text {concu }} & =\sum_{\left|\mathcal{C}_{i}\right| \neq 1} p_{i}^{*}\left(1-\prod_{j \in \mathcal{C}_{i} \backslash i}\left(1-p_{j}^{*}\right)\right) \prod_{k \in \mathcal{I}_{i}}\left(1-p_{k}^{*}\right) .
\end{aligned}
$$

The success rate of concurrent transmissions, $P_{\text {concu }}$, counts all the packet success rates of such senders: 1) the sender has at least one compatible sender, i.e., $\left|\mathcal{C}_{i}\right| \neq 1$, and 2) at least one compatible sender transmits a packet simultaneously with the sender, i.e., $p_{i}^{*}\left(1-\prod_{j \in \mathcal{C}_{i} \backslash i}\left(1-p_{j}^{*}\right)\right)$. Then the relation between the total packet success rate and its two components can be shown as $\sum_{i} u_{i}^{*}=P_{\text {single }}+P_{\text {concu }}$. Further, considering that simultaneous transmissions by incompatible senders cause packet collisions, we can also obtain the packet 
collision rate by

$$
P_{\text {colli }}=\sum_{i} p_{i}^{*}\left(1-\prod_{j \in \mathcal{I}_{i}}\left(1-p_{j}^{*}\right)\right)
$$

which can be further represented by $P_{\text {colli }}=\sum_{i} p_{i}^{*}-\sum_{i} u_{i}^{*}$.

In order to investigate how the optimal channel access probabilities in DAPMAC improve the network throughput, we first define two measured ratios: the concurrent ratio $\left(r_{\text {concu }}\right)$ and the collision ratio $\left(r_{\text {colli }}\right)$. The concurrent ratio is defined as the ratio between the success rate of concurrent transmissions $\left(P_{\text {concu }}\right)$ and the total packet success rate $\left(\sum_{i} u_{i}^{*}\right)$. The higher the concurrent ratio, the higher portion of concurrent transmissions in all successful transmissions. The collision ratio is defined as the ratio between the packet collision rate $\left(P_{\text {colli }}\right)$ and $\sum_{i} u_{i}^{*}$.

We then compare DAP-MAC to $p$-persistent slotted Aloha (S-ALOHA) 19] with its optimal access probability setting of $p=\frac{1}{N}$. As a quick note, considering the interference factor of node $i,\left|\mathcal{I}_{i}\right|$, is bounded by $N-1$, according to Theorem 1 the optimal access probability for node $i$ is $p_{i}^{*} \geq \frac{1}{N}$, always higher or equal to the ones predefined in S-ALOHA.

We take the network shown in Figure $2 \mathrm{a}$ as an example. Figure 5 depicts the optimal access probabilities and the distribution of packet success rates achieved by the senders. It can be seen that by considering the group compatibility relation, the optimal probabilities in DAP-MAC are scaled in accordance with individual sender's concurrent transmission capability, and the throughputs achieved by the senders are redistributed. We also find that the concurrent ratio $\left(r_{\text {concu }}\right)$ of DAP-MAC is 0.3 , approximately 1.7 times higher than S-ALOHA (0.18), while $r_{\text {colli }}$ is about $13 \%$ higher than S-ALOHA. The slightly higher collision ratio for DAP-MAC is caused by its more aggressive transmissions. However, compared to S-ALOHA, the total network throughput of DAP-MAC is still significantly improved, about 1.2 times higher than S-ALOHA.

Note that our utility-optimization framework considers proportional fairness. If the objective function is represented by the true throughput without fairness 350 constraint, i.e., $\widehat{U}(\mathrm{p})=\sum_{i} u_{i}$, it can be shown that its optimal transmission 


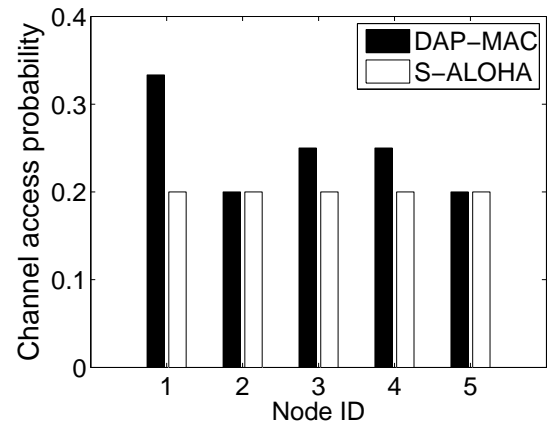

(a) Channel access probability.

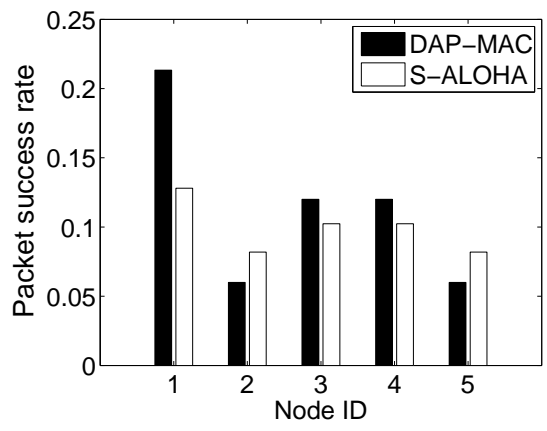

(b) Packet success rate.

Figure 5: Theoretical comparison of DAP-MAC and S-ALOHA in terms of channel access probability and packet success rate for the network shown in Figure $2 \mathrm{a}$

strategy is only to allow one of the existing compatible pairs to use the channel (e.g., nodes 1,3 or nodes 1,4 for the above example) and prohibit others from sending packets. Though the throughput for this case can be very high because of the exclusive channel access by a compatible pair, this is an unfair channel allocation which should be avoided in networking design. The proportional fairness considered in DAP-MAC allocates the channel access according to packet success rates of senders, which balances the throughput and fairness among competing senders.

\section{Performance Evaluation}

\subsection{Simulation Settings}

We evaluate our protocol through extensive simulations on the widely used network simulator OMNeT++-4.3 33 with INET-1.99.4 extension 34]. We implement the underwater acoustic communication link in the physical layer. We use an error-free channel model where packets propagate in the channel with a speed of $1500 \mathrm{~m} / \mathrm{s}$ and all lost packets are due to collisions and exceeding the maximum number of retransmissions (set at 10). We set the data transmission rate at $10 \mathrm{kbps}$, and the distance threshold $\Delta_{\text {Thresh }}$ at $160 \mathrm{~m}$. Data packets 
are generated following a Poisson process with an average rate of $\lambda$. Unless otherwise mentioned, all data packets are 128 bytes long and the MAC header is 4 bytes. All results are obtained by averaging 30 runs with each run lasting 2000 simulation seconds, and shown with $95 \%$ confidence.

We compare DAP-MAC with the following representative MAC protocols: p-persistent slotted Aloha (S-ALOHA) [19], slotted FAMA (S-FAMA) [4] and DOTS [5]. We use S-ALOHA as the handshaking-free baseline for comparison to show the advantage of dynamically assigned probabilities with concurrent transmissions considered in DAP-MAC. S-FAMA and DOTS are well-known underwater protocols which both use two-way handshaking. S-FAMA adopts the basic two-way handshaking in a time-slotted manner, while DOTS schedules parallel transmissions opportunistically via overhearing. We compare these two protocols with DAP-MAC to show how the system performance is impacted by handshaking as well as concurrent transmissions.

We evaluate the protocols under three types of networks: (a) A star network: shown in Figure 2a. We use it to demonstrate why DAP-MAC is able to improve the system throughput with concurrent transmissions in a probabilistic random access framework. (b) A static ad hoc network: we deploy 25 nodes in a $2000 \mathrm{~m} \times 2000 \mathrm{~m}$ horizontal square area in a $5 \times 5$ grid. We use this network to evaluate the performance of MAC protocols in typical multi-sender multi-receiver UWSNs. (c) A mobile ad hoc network: we randomly deploy 25 nodes in the same square area and allow nodes to move within a maximum range of $100 \mathrm{~m}$. The underwater mobility model we adopt is the kinematic model 35] for water currents composed of tides and eddies. We use this type of network to test the robustness of our protocol to network dynamics.

The following performance metrics are evaluated in the simulations: (a) Packet sending rate: the number of packets sent per second, which is a measure of channel occupation. (b) Number of retransmissions per successfully delivered packet: a direct measure of collisions which includes all retransmissions. (c) Throughput: the total number of successfully received data packets per second, which reflects how the channel bandwidth is utilized 
in a given amount of time. (d) Packet end-to-end delay: the average time

between a data packet's release time to the MAC layer and the time when it is successfully delivered at the receiver. It consists of queuing delay, transmission delay, and propagation delay. Propagation delay is defined as the packet travel time in the underwater channel. It can be calculated by the time it takes for the first bit of a data packet to travel from the sender to the receiver. A welldesigned MAC protocol should achieve high throughput with controlled packet delay.

\subsection{Simulation Results}

\subsubsection{The Star Network}

We first investigate how concurrent transmissions effectively improve the system throughput. We set $\lambda$ at $1 \mathrm{pkt} / \mathrm{s}$ as the saturated condition. Figure 6 presents the total number of successfully received packets by the receiver, and also among them the number of packets by single and concurrent transmissions. It is seen that the throughput improvement of DAP-MAC is mainly contributed by concurrent transmissions with the concurrent ratio 1.7 times higher than S-ALOHA. By taking advantage of nodes' locations and exploring the multisending opportunities, the throughput of DAP-MAC is greatly increased (also 1.2 times higher). Note that the total number of received packets in experiments is $8 \%$ lower than the theoretical value due to the channel usage by the control packets (e.g., ACK).

The performance metrics of the four protocols for the star network are evaluated in Figure 7. For all protocols the packet sending rates monotonically increase with $\lambda$ until leveling off from some points. S-FAMA is the worst in sending rates because of its exclusive channel access policy and the long handshaking delay. S-ALOHA is better without handshaking but suffer from limited channel access probability. DOTS shows a higher sending rate than DAP-MAC. However, as Figure $7 \mathrm{~b}$ shows, the high sending rate exceeds what the network can handle which results in lots of packet collisions.

Figure $7 \mathrm{~b}$ gives the number of packet retransmissions for each successfully 


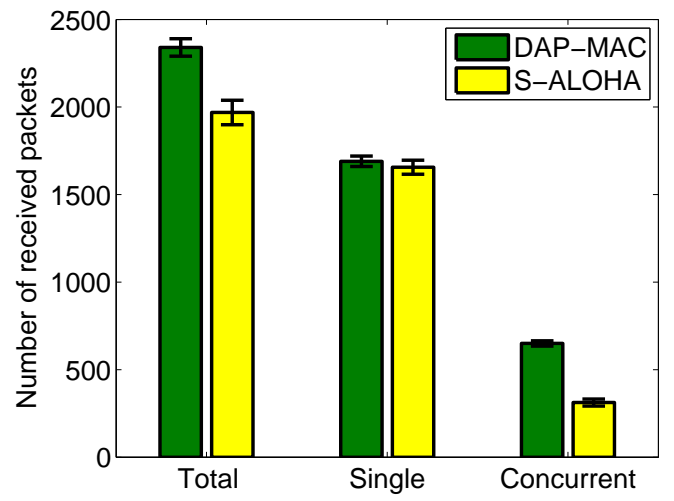

Figure 6: Star network: total number of successfully received packets and among them the number of packets by single and concurrent transmissions under the saturated condition $(\lambda=1 \mathrm{pkt} / \mathrm{s})$.

delivered packet. As the packet generation rate increases, S-FAMA keeps low retransmissions because of more reliable transmissions after handshaking, while DAP-MAC demonstrates slightly higher retransmissions than S-ALOHA as discussed in Section 4.2. DOTS exhibits the highest retransmissions due to large collisions on parallel transmissions, caused by failures to overhear the ongoing transmissions in hidden sender nodes.

Fig. $7 \mathrm{c}$ and $7 \mathrm{~d}$ present the throughput and packet end-to-end delay results, respectively. By keeping appropriate packet sending rates and relative fewer collisions, DAP-MAC is able to achieve the highest throughput among the four protocols, and the lowest packet delay at low data loads $(<0.4 \mathrm{pkts} / \mathrm{s})$. SFAMA has the lowest throughput due to low packet sending rate, but fewer collisions result in the smallest delay at high data loads. DOTS and S-ALOHA exhibit similar throughput performance and also similar delay (high). At high data loads, S-FAMA shows a bit lower delay than DAP-MAC. This is because when the channel bears high contention, the time spent in long handshaking is more rewarded by the reliable transmissions in terms of reducing the packet queuing delay. In this case, S-FAMA may be more suitable for the heavy traffic condition with delay-sensitive services. 


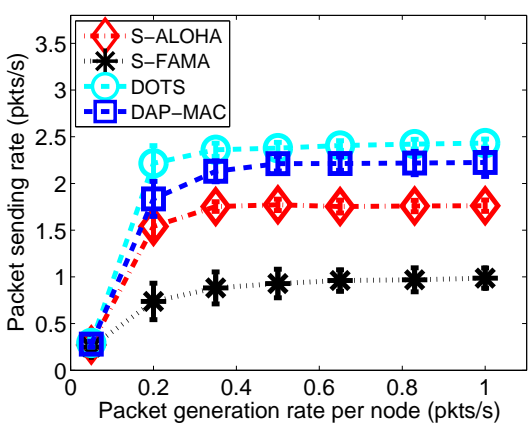

(a) Data packet sending rate.

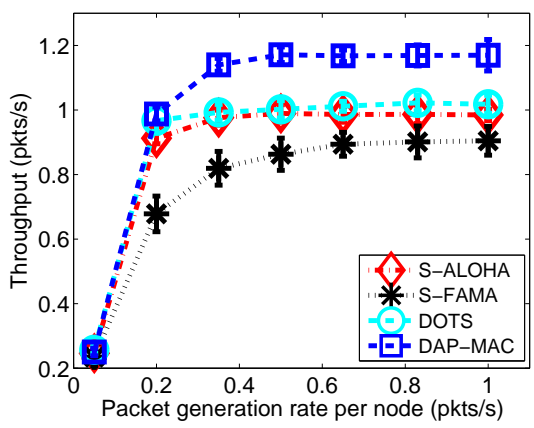

(c) Throughput.

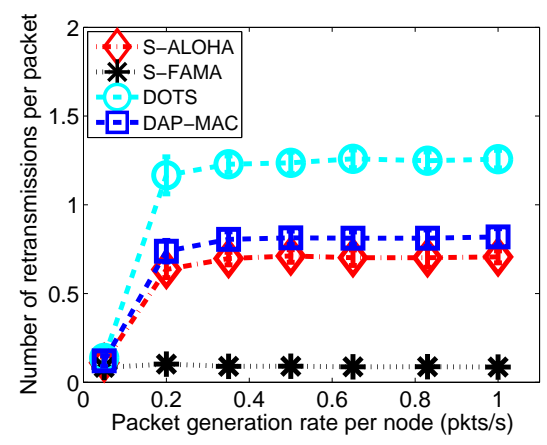

(b) Number of retransmissions.

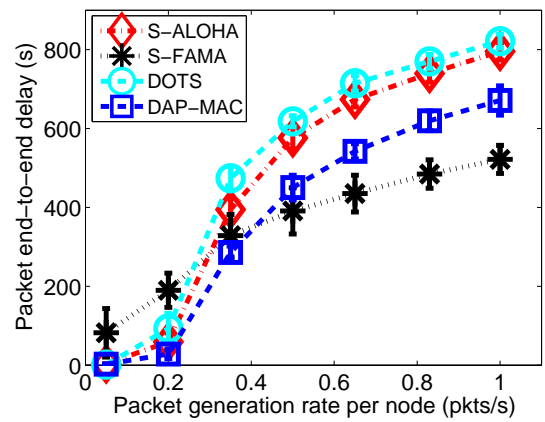

(d) Packet end-to-end delay.

Figure 7: Star network: packet sending rate, number of retransmissions per successfully delivered packet, throughput and packet end-to-end delay for the four protocols.

We next evaluate the impact of data packet size to the network throughput of the four protocols. We vary packet size from 64 bytes to 256 bytes and set $\lambda$ to $1 \mathrm{pkt} / \mathrm{s}$ as the saturated condition. Note that the slot size in DAP-MAC also changes with packet size as packet size affects the packet transmission time. Figure 8 shows the throughput results. It can be seen that as the packet size increases, the throughputs of all protocols decrease due to longer packet transmission time and lower packet sending rate. At smaller packet sizes, for example, 64 and 128 bytes, DAP-MAC demonstrates significantly higher throughput than the other protocols. This is because given a fixed data rate, packets with smaller sizes require shorter packet transmission time, resulting in a higher opportunity of successful concurrent transmissions. DAP-MAC is capable of capturing such 


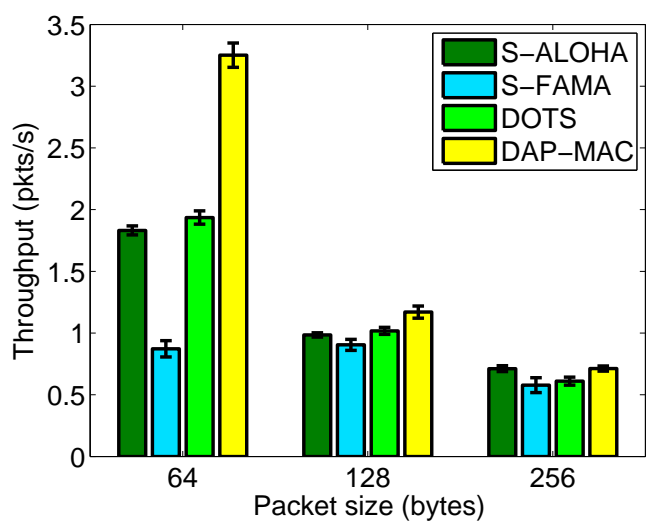

Figure 8: Star network: throughput performance for the four protocols with different data packet sizes ( 64 bytes, 128 bytes and 256 bytes) under the saturated condition $(\lambda=1 \mathrm{pkt} / \mathrm{s})$, which is used to show the impact of the packet size to the network throughput.

opportunity by optimally assigning higher access probabilities to senders who have more compatible nodes, so as to efficiently utilize the channel. In contrast, S-FAMA has the worst throughput due to its exclusive channel access policy, and S-ALOHA and DOTS are better than S-FAMA by scheduling limited concurrent transmissions. At a higher packet size (e.g., 256 bytes), longer packet transmission time reduces the chance of successful concurrent transmissions. In the studied network, no sender has compatible nodes and their optimal access probabilities equal $1 / N$, which is the channel access probability of S-ALOHA. Therefore, the two probabilistic protocols, S-ALOHA and DAP-MAC, achieve the similar throughput in this case.

\subsubsection{The Static Ad Hoc Network}

In a multi-sending multi-receiving ad hoc network, a node serves as both a sender and a receiver for different star subnetworks. The studied network, with nodes labeled from 1 to 25 row by row in a $5 \times 5$ grid, can be viewed as 25 overlapping star subnetworks. Three types of compatible graphs, classified by the positions of receivers (corner, side or inner), characterize the compatibility relations of these subnetworks. Figure 9 shows the examples of these graphs, 


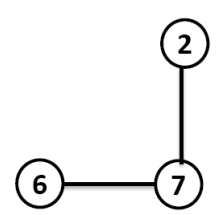

$p_{2}=p_{6}=1 / 2$

$p_{7}=1$

$\mathrm{E}[\mathrm{CF}]=7 / 3$

$\sum_{i} u_{i}=3 / 2$

(a) Corner.

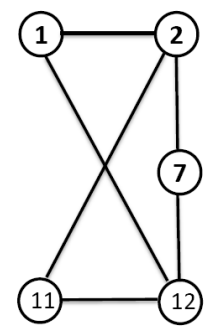

$p_{1}=p_{7}=p_{11}=1 / 3$

$p_{2}=p_{12}=1 / 2$

$E[C F]=17 / 5$

$\sum_{i} u_{i}=17 / 18$

(b) Side.

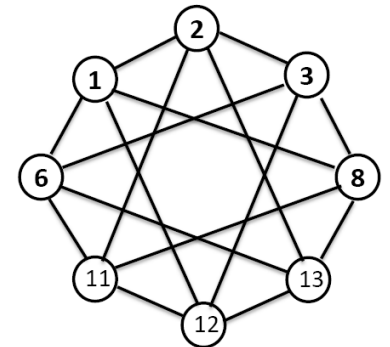

$p_{\mathrm{i}}=1 / 4$,

$i=1,2,3,6,8,11,12,13$

$\mathrm{E}[\mathrm{CF}]=5$

$\sum_{i} u_{i}=27 / 32$

(c) Inner.

Figure 9: Ad hoc network: three types of compatible graphs for the 25 overlapping star subnetworks, classified by the positions of receivers (corner, side or inner).

which represent the compatibility relations of corner node 1 , side node 6 and inner node 7, and these overlapping star subnetworks consist of 4 networks with graph (a), 12 with graph (b) and 9 with graph (c). The optimal channel access probabilities, the mean of CFs and the packet success rate $\left(\sum_{i} u_{i}\right)$ are also given for each graph (star network). We see that a receiver's average CF can be as high as 5 in graph (c), which implies a high opportunity of successful concurrent transmissions that can be scheduled by DAP-MAC.

We present the simulation results on the throughput and packet end-to-end delay of the ad hoc network in Figure 10, which shows similar throughput results as in Figure 7c As for the packet delay, DAP-MAC achieves the smallest delay at lower data loads, and has similar performance with the other three at higher loads. S-FAMA is the worst in packet delay due to its long handshaking.

We next estimate the throughput upper-bound of the overlapping star subnetworks in the studied ad hoc network, assuming no inter-network interference among the subnetworks and that every node always has packets to send. According to (8), by summing all the normalized packet success rates $\left(u_{i} / T_{s}\right)$ of the 25 subnetworks with each discounted by the different sending rates of the 


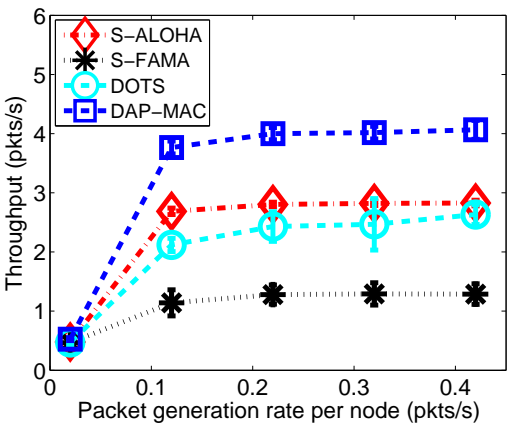

(a) Throughput.

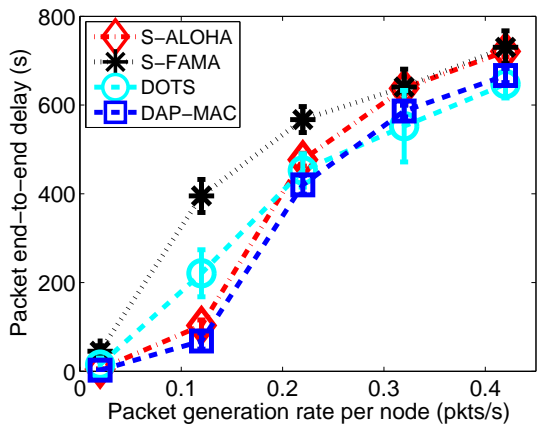

(b) Packet end-to-end delay.

Figure 10: Ad hoc network: throughput and packet end-to-end delay for the four protocols.

senders, we obtain the throughput upper-bound for the studied network as approximately $7 \mathrm{pkts} / \mathrm{s}$. It can be seen that the saturated throughput (4 pkts/s) achieved in the simulation, as shown in Figure 10a. is $40 \%$ lower than this upper bound. The reason for such throughput gap is due to the bandwidth usage by the control packets as well as the interferences caused by transmissions of nodes on the edges of the star subnetworks.

We further examine these interferences by removing some network connections to reduce interference and observing the throughput change. We create a new network, as shown in Figure 11, which is composed of four disjoint star subnetworks. The four networks are partitioned from the original network and the new network consists of the groups of one corner receiver 25, two side receivers 10 and 22, and one inner receiver 7. Other nodes are all senders, and packets are destined to their respective receivers with the packet generation rate unchanged. Compared to the original network, in the new network only the links associated with the four receivers are kept, and hence there are no DATA-DATA interferences and fewer DATA-ACK interferences. We compare the throughputs achieved at the four receivers for the two networks in Figure 12 . It can be seen that approximately $0.3 \mathrm{pkts} / \mathrm{s}(30 \%)$ throughput reduction in the original network is caused by the inter-network interferences, which include both 


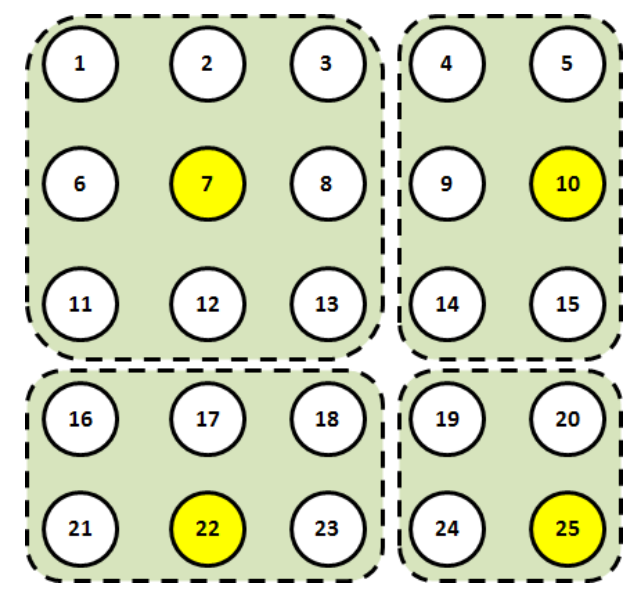

Figure 11: Ad hoc network: four networks are partitioned from the original network and the new network consists of the groups of one corner receiver 25, two side receivers 10 and 22, and one inner receiver 7 .

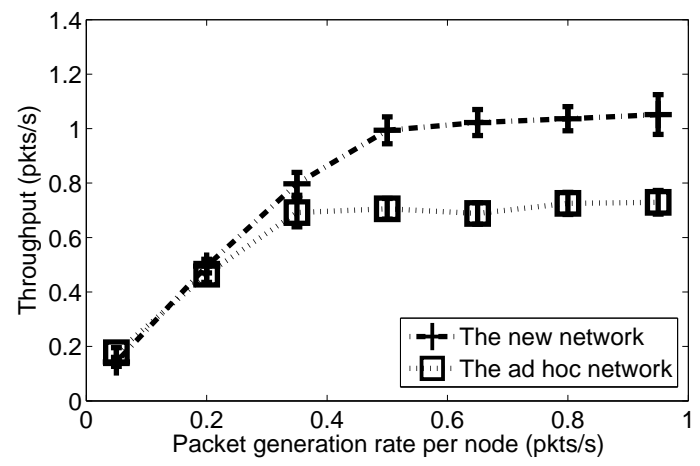

Figure 12: Ad hoc network: throughput performance between the new network and the original network, which is used to investigate the interference.

DATA-DATA and DATA-ACK interferences between the four subnetworks and others.

\subsubsection{Mobility Test}

We next examine the robustness of DAP-MAC under mobile underwater environment, where node mobility may cause link disruptions and result in data transmission failures. In the mobility testing, we set the update sending limit 


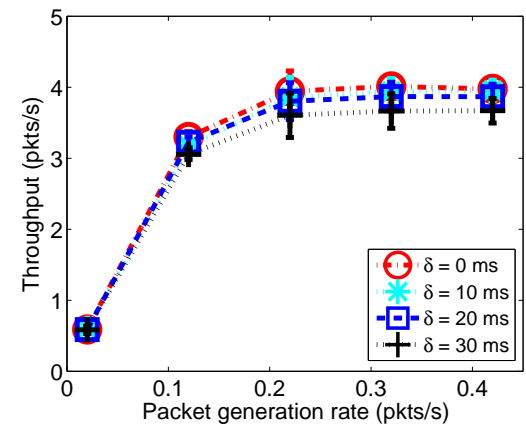

(a) Throughput.

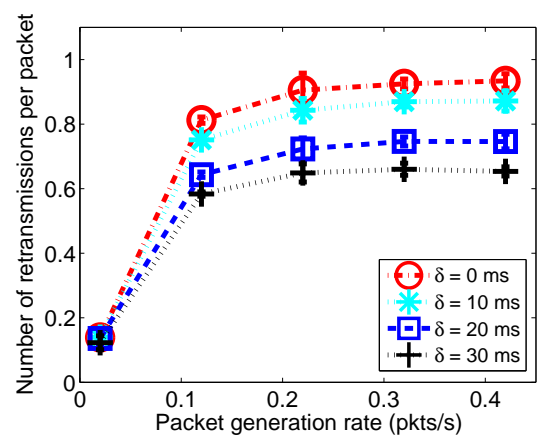

(b) Number of retransmissions.

Figure 13: Mobility testing: throughput and number of retransmissions per successfully delivered packets under different guard time settings: $0 \mathrm{~ms}, 10 \mathrm{~ms}$, $20 \mathrm{~ms}$ and $30 \mathrm{~ms}$.

( $T_{\text {update }}$ ) at $10 \mathrm{~s}$ (approximately 20 times longer than $T_{s}$ ) and $\lambda$ at $0.4 \mathrm{pkts} / \mathrm{s}$ by default. We adjust the node moving speed to control the link disruption time.

With node mobility, the distance variation between a sender and a receiver may result in a change on the group compatibility relation, and senders may not have the most updated compatibility information which leads to non-optimal channel access probabilities. In order to enhance robustness in the dynamic underwater networks, DAP-MAC adds a guard time interval $(\delta)$ in the compatibility threshold $\left(\Delta_{\text {thresh }}\right)$. The setting of the guard time is critical to system performance, as a shorter guard time interval promotes concurrent transmissions but may result in higher chance of packet collisions, while a longer guard time interval decreases the chance of unsuccessful concurrent transmissions but may lead to lower channel utilization. In Figure 13 , we show the throughput and packet retransmissions with the guard time interval varying from 0 to $30 \mathrm{~ms}$ at the node moving speed of $1.7 \mathrm{~m} / \mathrm{s}$. It can be seen that a shorter guard time interval achieves a bit higher throughput but causes more packet retransmissions, and vice versa. We can choose a guard time interval of $20 \mathrm{~ms}$ to balance the throughput and packet retransmissions.

We next observe how the compatibility factors of a receiver's neighbors change with time to show the opportunity of successful concurrent transmis- 


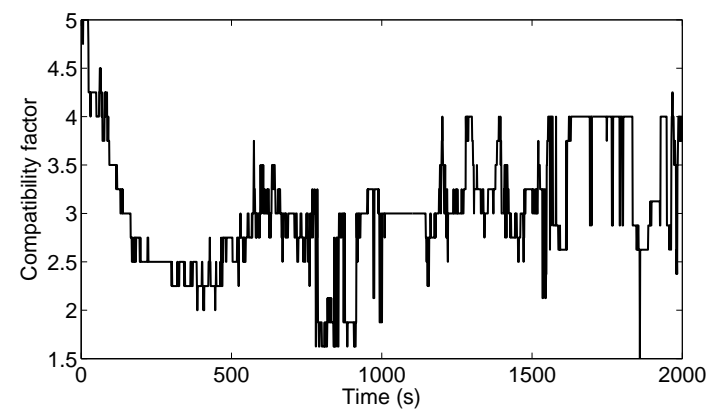

Figure 14: Mobility testing: variation of compatibility factor of an observation node with node moving speed of $1.7 \mathrm{~m} / \mathrm{s}$.

sions. We choose an observation node near the center of the network. We set the node moving speed at $1.7 \mathrm{~m} / \mathrm{s}$. As Figure 14 depicts, the average $\mathrm{CF}$ of the node's neighbors is above 1.5 for all the time instants and may go as high as 5, which indicates large opportunities for DAP-MAC to schedule successful concurrent transmissions and increase the channel utilization.

To examine how a node reacts to the compatibility relation changes and how fast the change is broadcast to its neighbors, we measure the average delay of a node sending UPDATE packets since the compatibility relation changes. The results are shown in Figure 15a, which also includes the average relation change intervals. It can be seen that with the speed increasing, changes occur more frequently. Under all the speeds, the change can be quickly recognized and broadcast to neighbors within the time that is only a small portion $(<1 / 5)$ of the real change intervals, which shows DAP-MAC's fast responsiveness to network dynamics.

The curves in Figure $15 \mathrm{~b}$ show the system throughput achieved by the four protocols varying with the node moving speed. We see that the handshakingbased protocols (S-FAMA and DOTS) are more vulnerable to nodes movement, especially under higher mobility. This is because the two-way handshaking requires two propagation delays and therefore the probability of link disruptions in each round of data transmission is twice that of handshaking-free probability- 


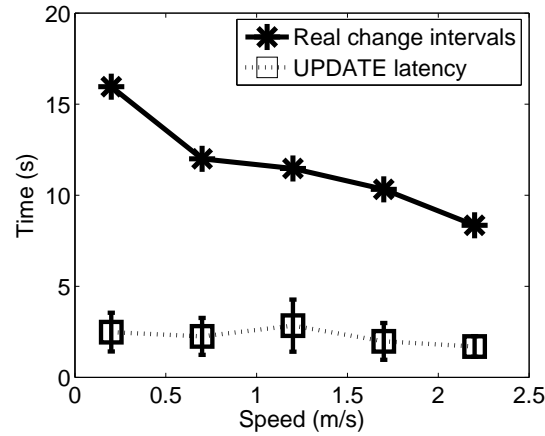

(a) UPDATE sending latency.

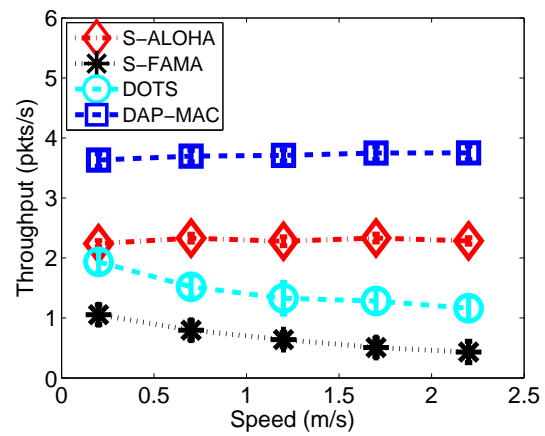

(b) Throughput.

Figure 15: Mobility testing: (a) UPDATE sending latency of a node sending UPDATE packets since the compatibility relation changes. (b) Throughput comparison for the four protocols.

based protocols. As the mobility becomes higher, link disruptions are more likely to happen. In contrast, the probability-based protocols schedule data transmissions much faster and they are less vulnerable to dynamics. With the mobility support, DAP-MAC is able to schedule successful concurrent transmissions to achieve higher throughput over the other protocols.

\section{Conclusion and Future Work}

In this paper, we propose a delay-aware probability-based random access protocol, DAP-MAC, for long-delay underwater sensor networks. The protocol leverages the long acoustic propagation delay for concurrent sending with prescribed optimal transmission probabilities. The probabilities are obtained distributively at run-time by a utility optimization framework to maximize the overall throughput considering the group compatibility relation of senders. Significant advantage of DAP-MAC in terms of system throughput, packet end-toend delay, and robustness to network dynamics has been demonstrated by our simulation results over other typical underwater MAC protocols.

Our proposed DAP-MAC framework currently does not consider the multipath effect. However, the protocol can be extended to con- 
sider it should it arise in scenarios like shallow water environment. Currently the optimal channel access probability for a sender is obtained based on its packet success capability, which would be an overestimation under severe multipath effect because the multipath effect will result in an increase of both the packet error rate and packet collision rate. DAP-MAC needs to incorporate the more elaborated UWSN channel model at the physical layer to adjust the optimal transmission strategy accordingly for throughput optimization.

\section{Acknowledgments}

This work was supported in part by Office of Naval Research under grants N00014-10-1-0762 and N00014-12-1-0070 and National Science Foundation under grant MRI-1428567.

\section{References}

[1] I. F. Akyildiz, D. Pompili, T. Melodia, Underwater acoustic sensor networks: Research challenges, Ad Hoc Netw. 3 (3) (2005) 257-279.

[2] J. Heidemann, M. Stojanovic, M. Zorzi, Underwater sensor networks: Applications, advances, and challenges, Philosophical Transactions of the Royal Society-A 370 (1958) (2012) 158-175.

[3] M. Stojanovic, Underwater acoustic communication, Wiley Encyclopedia of Electrical and Electronics Engineering 22 (1998) 688-698.

[4] M. Molins, M. Stojanovic, Slotted FAMA: a MAC protocol for underwater acoustic networks, in: Proc. IEEE OCEANS, 2006, pp. 1-7.

[5] Y. Noh, P. Wang, U. Lee, D. Torres, M. Gerla, DOTS: A propagation delay-aware opportunistic MAC protocol for underwater sensor networks, in: Proc. IEEE ICNP, 2010, pp. 183-192. 
[6] C. Petrioli, R. Petroccia, M. Stojanovic, A comparative performance evaluation of MAC protocols for underwater sensor networks, in: Proc. IEEE OCEANS, 2008, pp. 1-10.

[7] B. Peleato, M. Stojanovic, Distance aware collision avoidance protocol for ad-hoc underwater acoustic sensor networks, IEEE Commun. Lett. 11 (12) (2007) 1025-1027.

[8] L. Massoulie, J. Roberts, Bandwidth sharing: objectives and algorithms, IEEE/ACM Trans. Netw. 10 (3) (2002) 320-328.

[9] J.-W. Lee, M. Chiang, A. Calderbank, Utility-optimal random-access control, IEEE Trans. Wireless Commun. 6 (7) (2007) 2741-2751.

[10] J. Liu, Y. Yi, A. Proutiere, M. Chiang, H. V. Poor, Towards utility-optimal random access without message passing, Wirel. Commun. Mob. Comput. 10 (2010) 115-128.

[11] K. Kar, S. Sarkar, L. Tassiulas, Achieving proportional fairness using local information in aloha networks, IEEE Trans. Autom. Control 49 (10) (2004) $1858-1863$.

[12] J. Yackoski, C.-C. Shen, UW-FLASHR: Achieving high channel utilization in a time-based acoustic MAC protocol, in: Proc. ACM WUWNet, 2008, pp. 59-66.

[13] C.-C. Hsu, K.-F. Lai, C.-F. Chou, K. Lin, ST-MAC: Spatial-temporal MAC scheduling for underwater sensor networks, in: Proc. IEEE INFOCOM, 2009, pp. 1827-1835.

[14] K. Kredo, P. Djukic, P. Mohapatra, STUMP: Exploiting position diversity in the staggered TDMA underwater MAC protocol, in: Proc. IEEE INFOCOM, 2009, pp. 2961-2965.

[15] X. Guo, M. Frater, M. Ryan, An adaptive propagation-delay-tolerant MAC protocol for underwater acoustic sensor networks, in: Proc. IEEE OCEANS, 2007, pp. 1-5. 
[16] Z. Peng, Y. Zhu, Z. Zhou, Z. Guo, J.-H. Cui, COPE-MAC: A contentionbased medium access control protocol with Parallel Reservation for underwater acoustic networks, in: Proc. IEEE OCEANS, 2010, pp. 1-10.

[17] N. Chirdchoo, W.-S. Soh, K. Chua, RIPT: A receiver-initiated reservationbased protocol for underwater acoustic networks, IEEE J. Sel. Areas Commun. 26 (9) (2008) 1744-1753.

[18] T. Hu, Y. Fei, DSH-MAC: Medium access control based on decoupled and suppressed handshaking for long-delay underwater acoustic sensor networks, in: Proc. IEEE LCN, 2013, pp. 523-531.

[19] D. Bertsekas, R. Gallager, Data Networks, Prentice Hall, New Jersey, 1992.

[20] A. A. Syed, W. Ye, J. Heidemann, B. Krishnamachari, Understanding spatio-temporal uncertainty in medium access with aloha protocols, in: Proc. ACM WUWNet, 2007, pp. 41-48.

[21] N. Chirdchoo, W.-S. Soh, K. C. Chua, Aloha-based mac protocols with collision avoidance for underwater acoustic networks, in: Proc. IEEE INFOCOM, 2007, pp. 2271-2275.

[22] Y. Zhou, K. Chen, J. He, H. Guan, Enhanced slotted aloha protocols for underwater sensor networks with large propagation delay, in: Proc. IEEE VTC Spring 2011, 2011, pp. 1-5.

[23] P. Mandal, S. De, S. S. Chakraborty, A receiver synchronized slotted aloha for underwater wireless networks with imprecise propagation delay information, Ad Hoc Netw. 11 (4) (2013) 1443-1455.

[24] A. Syed, W. Ye, J. Heidemann, T-Lohi: A new class of MAC protocols for underwater acoustic sensor networks, in: Proc. IEEE INFOCOM, 2008, pp. 231-235.

[25] M. K. Park, V. Rodoplu, UWAN-MAC: An energy-efficient MAC protocol for underwater acoustic wireless sensor networks, IEEE J. Ocean. Eng. 32 (3) (2007) 710-720. 
[26] B. Chen, D. Pompili, Reliable geocasting for random-access underwater acoustic sensor networks, Ad Hoc Networks 21 (2014) 134 - 146.

[27] A. Syed, W. Ye, J. Heidemann, Time synchronization for high latency acoustic networks, in: Proc. IEEE INFOCOM, 2006, pp. 1-12.

[28] N. Chirdchoo, W.-S. Soh, K. C. Chua, MU-Sync: A time synchronization protocol for underwater mobile networks, in: Proc. ACM WUWNet, 2008, pp. $35-42$.

[29] H. Karl, A. Willig, Principles of Naval Weapons Systems, Kendall Hunt Pub Co, 2000.

[30] X. Lurton, An Introduction to Underwater Acoustics: Principles and Applications, Geophysical Sciences Series, Springer, 2002.

URL http://books . google.com/books?id=VTNRh3pyCyMC.

[31] I. Chakeres, E. Belding-Royer, The utility of hello messages for determining link connectivity, in: Proc. Int. Symp. Wireless Personal Multimedia Commun., Vol. 2, 2002, pp. 504-508.

[32] H. Stark, J. W. Woods, Probability, Statistics, and Random Processes for Engineers, Prentice Hall, 2002.

[33] Andras Varga and OpenSim Ltd., OMNeT++ User Manual, 2013.

URL http://www. omnetpp.org

[34] INET Framework Manual, 2012.

URL http://inet.omnetpp.org

[35] S. P. Beerens, H. Ridderinkhof, J. Zimmerman, An analytical study of chaotic stirring in tidal areas, Chaos, Solitons and Fractals 4 (6) (1994) $1011-1029$. 